\title{
An exact effective Hamiltonian for a perturbed Landau level
}

\author{
Michael Wilkinson $\dagger$ \\ Department of Physics, 405-47 California Institute of Technology, Pasadena, CA 91125 , \\ USA and Instituto de Fisica Gleb Wataghin, Universidade Estadual de Campinas, \\ Campinas, $13100 \mathrm{SP}$, Brazil
}

Received 17 July 1986

\begin{abstract}
This paper considers the effect of a scalar potential $V(x, y)$ on a Landau level in two dimensions. An exact effective Hamiltonian is derived which describes the effect of the potential on a single Landau level, expressed as a power series in $V / E_{c}$, where $E_{c}$ is the cyclotron energy.

The effective Hamiltonian can be represented as a function $H(x, p)$ in a onedimensional phase space. The function $H(x, p)$ resembles the potential $V(x, y)$ : when the area of a flux quantum is much smaller than the square of the characteristic length scale of $V$, then $H \simeq V$. Also $H(x, p)$ retains the translational and rotational symmetries of $V(x, y)$ exactly, but reflection symmetries are not retained beyond the lowest order of the perturbation expansion.
\end{abstract}

\section{Introduction}

The perturbation of a Landau level in a two-dimensional system by a scalar potential is a problem of considerable theoretical interest. There are two reasons for this. Firstly, an analysis of this problem is essential to understanding the quantised Hall effect. Secondly, when the potential is spatially periodic, this is an example of the problem of Bloch electrons in a magnetic field, which has many interesting mathematical properties: for instance, it is believed that the spectrum is typically a Cantor set.

This paper will derive an exact effective Hamiltonian which describes the effect of the potential $V(x, y)$ on a given Landau level, expressed as a power series in $V / E_{\mathrm{c}}$, where $E_{\mathrm{c}}=h e B / m$ is the cyclotron energy. The effective Hamiltonian $H_{\mathrm{eff}}^{(n)}$ of the $n$th Landau level is expressed as an operator in a one-dimensional phase space: $\hat{H}_{\mathrm{eff}}^{(n)}=$ $H^{(n)}(\hat{x}, \hat{p})$. This representation is both useful, because the original two-dimensional problem has been reduced to a problem in one dimension, and also intuitively appealing because the function $H(x, p)$ resembles the potential $V(x, y)$. A natural dimensionless parameter is $\beta$, the area of a flux quantum divided by the square of the characteristic length scale of the potential; when the potential is periodic we can define $\beta$ precisely as the number of unit cells per flux quantum. When $\beta \rightarrow 0$, the Hamiltonian function tends towards the potential: $H \rightarrow V$.

Even when $\beta$ is not small, there are still similarities between $H$ and $V$, because $H$ retains any translational and rotational symmetries of the potential. Reflection sym. metries of $V$ are also retained in $H$ in the first order of perturbation theory.

\footnotetext{
† Permanent address: Department of Physics and Applied Physics, John Anderson Building, University of Strathclyde, Glasgow G4 0NG, UK.
} 
The preservation of symmetries of $V$ by the effective Hamiltonian $H$ is very important for the problem of Bloch electrons in a magnetic field. In this problem, it is believed that when $\beta$ is an irrational number, the spectrum is a Cantor set (Simon 1982), and perturbation theory suggests that this Cantor set should have finite measure. Numerical experiments have been done using one-dimensional effective Hamiltonians which are periodic functions of $x$ and $p$. These experiments show that when the effective Hamiltonian has centres of threefold or fourfold rotational symmetry in the phase plane, the spectrum is a Cantor set of zero measure for irrational values of $\beta$, with a remarkable hierarchical structure (Hofstadter 1976, Claro and Wannier 1979, Wilkinson 1984). The results of this paper show that this phenomenon is not just an artefact of the model, since effective Hamiltonians with exact threefold and fourfold symmetry in phase space result from potentials with threefold and fourfold axes in coordinate space, where these are natural crystallographic symmetries.

Section 2 of this paper evaluates the matrix elements of the perturbing potential in a basis of Landau states. By ignoring matrix elements coupling different Landau levels, the first-order approximation to $\hat{H}_{\text {eff }}$ is obtained. Section 3 shows how the interband matrix elements coupling different Landau levels can be eliminated, leading to an exact effective Hamiltonian. Section 4 discusses the preservation of symmetries of $V(x, y)$ in the effective Hamiltonian $H(x, p)$. In several places a linear canonical transformation will be used. Canonical transformations create difficulties in quantum mechanical problems because they are usually ambiguous. If the transformation is linear, and the Hamiltonian is expressed as a Fourier integral, then there is no ambiguity however. The reasons for this are discussed in appendix 1.

The problem the perturbation of a Landau level by a periodic potential has been discussed in earlier papers by Rauh $(1974,1975)$ and Schellnhuber et al (1981). These authors expressed the effective Hamiltonian as a difference equation with periodic coefficients. This is essentially equivalent to quantising the effective Hamiltonian $H(x, p)$ in the position representation (Wilkinson 1984), but has the disadvantage that the symmetry of $H(x, p)$ is obscured, as well as the fact that $H \rightarrow V$ when $\beta$ is small. These papers also only give an approximate effective Hamiltonian, since they do not show how to eliminate matrix elements coupling different Landau levels.

\section{Representation in a basis of Landau states}

In this section the Hamiltonian will be expressed in a basis of Landau states $|n, q\rangle$, where $n$ labels the Landau level and $q$ is the internal coordinate for the $n$th Landau level. The first-order approximation to the effective Hamiltonian is obtained by ignoring matrix elements coupling different Landau levels.

First we introduce the Landau level basis. The Hamiltonian of our problem is

$$
\hat{H}=\frac{1}{2 m}(\hat{\boldsymbol{p}}-e \boldsymbol{A}(\hat{\boldsymbol{r}}))^{2}+V(\hat{\boldsymbol{r}})=\hat{H}_{0}+\hat{\boldsymbol{V}}
$$

and we will use the Landau gauge, $\boldsymbol{A}=(0, B x, 0)$. It is convenient to rescale the coordinates and momenta

$$
\boldsymbol{p} \rightarrow \boldsymbol{p}^{\prime}=\frac{1}{\sqrt{m}} \boldsymbol{p} \quad \boldsymbol{r} \rightarrow \boldsymbol{r}^{\prime}=\frac{e B}{\sqrt{m}} \boldsymbol{r}
$$

so that $\left[\hat{\boldsymbol{r}}^{\prime}, \hat{\boldsymbol{p}}^{\prime}\right]=i \hbar \omega_{c}$, where $\omega_{c}=e B / m$ is the cyclotron frequency. Note that the unperturbed Hamiltonian $\hat{H}_{0}$ is a quadratic function of the coordinates and momenta. 
Using a canonical transformation $H_{0}$ can be expressed in normal form; the generating function of this canonical transformation to a new set of coordinates $\left(q, q^{\prime}\right)$ and momenta $\left(p, p^{\prime}\right)$ is

$$
S=S\left(x, y, p, p^{\prime}\right)=x p^{\prime}+y p-p^{\prime} p .
$$

Using the relations

$$
p_{x}=\partial S / \partial x \quad p_{y}=\partial S / \partial y \quad q=\partial S / \partial p \quad q^{\prime}=\partial S / \partial p^{\prime}
$$

we find

$$
\begin{array}{ll}
p_{x}=p^{\prime} & x=q^{\prime}+p \\
p_{y}=p & y=q+p^{\prime} .
\end{array}
$$

Since the canonical transformation is linear, we have

$$
\hat{H}_{0}=\frac{1}{2}\left(\hat{p}^{\prime 2}+\hat{q}^{\prime 2}\right)
$$

(see appendix 1 ). Note that $\hat{H}_{0}$ is independent of $\hat{q}$ and $\hat{p}$. We can write the eigenstates of (2.6) in the form

$$
|n, q\rangle=|n\rangle \otimes|q\rangle
$$

where $|n\rangle$ is an eigenfunction of the harmonic oscillator Hamiltonian $\hat{H}_{0}$, and $|q\rangle$ is an eigenstate of the position operator $\hat{q}$, and these states are called Landau states. The energy of the $n$th Landau level is

$$
E_{n}=2 \pi\left(n+\frac{1}{2}\right) \hbar \omega_{\mathrm{c}}
$$

and its degeneracy per unit area is

$$
N=e B / h \text {. }
$$

Having introduced the Landau state basis, we can now take matrix elements of the potential $\hat{V}$ in this basis. Again it will be convenient to rescale some quantities: we write

$$
u(r / \sqrt{A})=V(r)
$$

where $A$ is the square of the characteristic length scale of the potential, or in the periodic case, the area of the unit cell. The function $u(r)$ then varies on a length scale of order unity. We also rescale the conjugate variables $q, p$ which form the internal coordinates of the Landau levels: write

$$
X=\frac{\sqrt{m}}{e B \sqrt{A}} p \quad P=-\frac{\sqrt{m}}{e B \sqrt{A}} q
$$

so that

$$
[\hat{X}, \hat{P}]=\mathrm{i} \hbar^{*} \quad \hbar^{*}=\hbar / e B A .
$$

The dimensionless quantity $\beta=2 \pi \hbar^{*}$ measures the ratio of the area of a flux quantum to the area of a unit cell (or some other characteristic area, if the potential is not periodic). Collecting together $(2.2),(2.5),(2.10)$ and (2.11), we have

$$
V=u\left(\frac{\sqrt{m}}{e B \sqrt{A}} q^{\prime}-X, \frac{\sqrt{m}}{e B \sqrt{A}} p^{\prime}-P\right) .
$$


The correct way to quantise this expression is to express the function $u$ as a Fourier integral

$$
u(\boldsymbol{r})=\int \mathrm{d} \boldsymbol{k} \tilde{u}(\boldsymbol{k}) \exp (\mathrm{i} \boldsymbol{k} \cdot \boldsymbol{r})
$$

(see appendix 1), so that

$$
\hat{V}=\int \mathrm{d} \boldsymbol{k} \exp \left(\frac{\mathrm{i} \sqrt{m}}{e B \sqrt{A}}\left(k_{x} \hat{q}^{\prime}+k_{y} \hat{p}^{\prime}\right)\right) \tilde{u}(\boldsymbol{k}) \exp \left[\mathrm{i}\left(k_{x} \hat{X}-k_{y} \hat{P}\right)\right] .
$$

We can therefore write the Hamiltonian in the form

$$
\hat{H}=\sum_{n n^{\prime}}|n\rangle\left\langle n^{\prime}\right|\left(2 \pi\left(n+\frac{1}{2}\right) \frac{e B}{m} \hbar \delta_{n n^{\prime}}+\int \mathrm{d} \boldsymbol{k} v_{n n^{\prime}}(\boldsymbol{k}) \exp \left[\mathrm{i}\left(k_{x} \hat{X}-k_{y} \hat{P}\right)\right]\right)
$$

where

$$
\begin{aligned}
v_{n n} \cdot(\boldsymbol{k}) & =\left\langle n\left|\exp \left(\frac{\mathrm{i} \sqrt{m}}{e B \sqrt{A}}\left(k_{x} \hat{q}^{\prime}+k_{y} \hat{p}^{\prime}\right)\right)\right| n^{\prime}\right\rangle \tilde{u}(\boldsymbol{k}) \\
& =\left\langle n\left|\exp \left(\frac{\mathrm{i} \hbar{ }^{* 1 / 2}}{\left(\hbar \omega_{\mathrm{c}}\right)^{1 / 2}}\left(k_{x} \hat{q}^{\prime}+k_{y} \hat{p}^{\prime}\right)\right)\right| n^{\prime}\right\rangle \tilde{u}(\boldsymbol{k}) .
\end{aligned}
$$

In (2.16), the terms in the large round brackets are the matrix elements of $\hat{H}$ between the Landau level indices $n, n^{\prime}$, which have been left in operator form with respect to the internal degree of freedom of the Landau levels $(\hat{X}, \hat{P})$. The functions of $\hat{X}, \hat{P}$ appearing in (2.16) have a characteristic length scale of order unity. The matrix element multiplying $\tilde{u}(\boldsymbol{k})$ in (2.17) tends to $\delta_{n n^{\prime}}$ as $\hbar^{*} \rightarrow 0$, with $\boldsymbol{k}$ of order unity. In fact this matrix element can be evaluated exactly:

$$
\begin{aligned}
\left\langle n\left|\exp \left[\mathrm{i}\left(\frac{\hbar^{*}}{\hbar \omega_{\mathrm{c}}}\right)^{1 / 2}\left(k_{z} \hat{q}^{\prime}+k_{y} \hat{p}^{\prime}\right)\right]\right| n^{\prime}\right\rangle & \\
= & \exp \left[\mathrm{i} \alpha\left(\theta-\frac{\pi}{2}\right)\right]\left(\frac{\hbar^{*} k^{2}}{2}\right)^{\alpha / 2} \exp \left(-\frac{1}{4} k^{2} h^{*}\right) \\
& \times\left(\frac{N !}{(N+\alpha) !}\right)^{1 / 2} L_{N}^{(\alpha)}\left(\frac{k^{2} h^{*}}{2}\right) \quad \alpha=\left|n-n^{\prime}\right|, N=\min \left(n, n^{\prime}\right)
\end{aligned}
$$

where $(k, \theta)$ are the polar coordinates of $\boldsymbol{k}=\left(k_{x}, k_{y}\right)$ with $\theta$ measured clockwise from the $k_{x}$ axis, and $L_{N}^{(\alpha)}$ is an associated Laguerre polynomial (see appendix 2).

Equations (2.16)-(2.18) and (2.12) contain the principal results of this section. A lowest order approximation to the effective Hamiltonian can be obtained by ignoring the off-diagonal matrix elements in (2.16):

$$
\hat{H}_{\mathrm{eff}}^{(n)}=\int \mathrm{d} \boldsymbol{k} v_{n n}(\boldsymbol{k}) \exp \left[\mathrm{i}\left(k_{x} \hat{X}-k_{y} \hat{P}\right)\right]+\left(n+\frac{1}{2}\right) h \omega_{\mathrm{c}} .
$$

This is equivalent to simply applying degenerate perturbation theory to the $n$th Landau level. For the problem of Bloch electrons in a magnetic field the integral in (2.19) is replaced by a summation, because $V(\boldsymbol{r})$ is periodic:

$$
H_{\mathrm{eff}}^{(n)}=\sum_{k} v_{n n}(\boldsymbol{k}) \exp \left[\mathrm{i}\left(k_{x} \hat{X}-k_{y} \hat{P}\right)\right]
$$


This effective Hamiltonian has all the geometrical symmetries of the potential $V(\boldsymbol{r})$ including symmetries under reflection. From (2.18), we see that in the semiclassical limit, $\hbar^{*} \rightarrow 0$, the effective Hamiltonian is the same as the scaled potential, $u(r)$ (see $(2.10))$ :

$$
H_{\mathrm{eff}}^{(n)} \underset{n^{*} \rightarrow 0}{\longrightarrow} u(\hat{X},-\hat{P})
$$

This fact has a simple interpretation. When $\hbar^{*}$ is small, the cyclotron radius is much smaller than the scale size of the potential, and this potential acts on the electron like a uniform electric field, equal to $\nabla u$. In a uniform electric field the electron drifts along an equipotential line at a rate proportional to $|\nabla u|$. This law of motion is exactly the same as Hamilton's equations in one dimension, with a Hamiltonian proportional to $u$.

\section{An exact effective Hamiltonian}

In this section the approximate effective Hamiltonian (2.19) will be made exact using a projection operator method.

When the perturbation $\hat{V}$ is applied, the Landau levels are broadened, but for small enough $\hat{V}$ the gaps in the spectrum between successive Landau levels do not close. Let $\hat{P}^{(n)}$ be the projection operator for the $n$th perturbed Landau level:

$$
\hat{P}^{(n)}=f(\hat{H}+\hat{V})
$$

with $f(E)$ equal to 1 if $E$ is in the broadened spectrum of the $n$th Landau level, and zero if $E$ is in any other part of the spectrum of $\hat{H}$. This projection operator is well defined so long as the gaps between the Landau levels remain open.

Consider the set of states obtained by projecting the Landau states $|n, q\rangle$ into the $n$th (perturbed) Landau level

$$
\left|n, q^{*}\right\rangle=\hat{P}^{(n)}|n, q\rangle \text {. }
$$

This set of states is independent of the eigenstates outside the $n$th Landau level, and provided they are linearly independent they form a complete basis for the $n$th Landau level. It will become clear that these states are linearly independent for sufficiently small values of $V$. The Schrödinger equation describing the $n$th perturbed Landau level is therefore

$$
\int \mathrm{d} q^{\prime}\left[\left\langle n, q\left|\hat{P}^{(n)} \hat{H} \hat{P}^{(n)}\right| n, q^{\prime}\right\rangle-E\left\langle n, q\left|\hat{P}^{(n)}\right| n, q^{\prime}\right\rangle\right]\left\langle n, q^{\prime} \mid \psi\right\rangle=0 .
$$

The condition for the states $\left|n, q^{*}\right\rangle$ to the linearly independent is that the normalisation matrix $\left\langle n, q\left|\hat{P}^{(n)}\right| n, q^{\prime}\right\rangle$ appearing in (3.3) should be non-singular. Equation (3.3) can be written symbolically in the form

$$
\left(\hat{H}^{(n) *}-E \hat{P}^{(n) *}\right)|\psi\rangle=0 .
$$

If $\hat{P}^{(n) *}$ is non-singular, as has been assumed, this equation can be written

$$
\left(\hat{H}_{\mathrm{eff}}^{(n)}-E\right)|\Psi\rangle=0
$$

where

$$
\hat{H}_{\mathrm{eff}}^{(n)}=\left(\hat{P}^{(n) *}\right)^{-1 / 2} \hat{H}^{(n) *}\left(\hat{P}^{(n) *}\right)^{-1 / 2}
$$

is the desired effective Hamiltonian. 
In order to evaluate (3.6) it is necessary to obtain an explicit expression for the projection operator. The most useful starting point is the equation

$$
\hat{P}=\frac{1}{2 \pi \mathrm{i}} \oint_{C} \mathrm{~d} E \hat{G}(E)
$$

where $\hat{G}=(\hat{H}-E)^{-1}$ is the Green function and $C$ is any contour which encloses only the required part of the spectrum. The Green function can be obtained from the Born formula

$$
\hat{G}(E)=\hat{G}_{0}(E)+\hat{G}_{0}(E) \hat{V} \hat{G}(E)
$$

by iteration; $\hat{G}_{0}(E)$ is the Green function for the unperturbed Landau levels:

$$
\begin{aligned}
\hat{G}_{0}(E) & =\int \mathrm{d} q \sum_{n} \frac{|n, q\rangle\langle n, q|}{E-E_{n}}=\sum_{n} \frac{|n\rangle\langle n|}{E-E_{n}} \int \mathrm{d} q|q\rangle\langle q| \\
& =\hat{1}^{*} \sum_{n} \frac{|n\rangle\langle n|}{E-E_{n}} .
\end{aligned}
$$

This leads to an expression for $\hat{P}^{(n)}$ in the form of a power series in $V$; the term of order $V^{N}$ will be written $\hat{P}_{N}^{(n)}$.

Using (3.7)-(3.9) and (2.16), we find

$$
\hat{P}_{0}^{(n)}=\int \mathrm{d} q|q\rangle\langle q|\otimes| n\rangle\langle n|=| n\rangle\langle n| \hat{\imath}^{*}
$$

where $\hat{1}^{*}$ is the identity operator for the internal coordinate and

$$
\begin{aligned}
\hat{P}_{1}^{(n)}=\sum_{m} \sum_{m^{\prime}} \iint \mathrm{d} q \mathrm{~d} q^{\prime}|m, q\rangle\left\langle m^{\prime}, q^{\prime}\right| \frac{\left\langle m, q|\hat{V}| m^{\prime}, q^{\prime}\right\rangle\left(\delta_{n m}-\delta_{n m^{\prime}}\right)}{h \omega_{c}\left(m-m^{\prime}\right)} \\
=\sum_{m} \sum_{m^{\prime}}|m\rangle\left\langle m^{\prime}\right| \frac{V_{n m^{\prime}}(\hat{X}, \hat{P})}{h \omega_{c}\left(m-m^{\prime}\right)}\left(\delta_{m n}-\delta_{m^{\prime} n}\right) \\
=\sum_{n^{\prime} \neq n}\left(|n\rangle\left\langle n^{\prime}\left|\frac{V_{n n^{\prime}}(\hat{X}, \hat{P})}{h \omega_{c}\left(n-n^{\prime}\right)}-\right| n^{\prime}\right\rangle\langle n| \frac{V_{n^{\prime} n}(\hat{X}, \hat{P})}{h \omega_{c}\left(n-n^{\prime}\right)}\right)
\end{aligned}
$$

where

$$
V_{n n^{\prime}}(\hat{X}, \hat{P})=\int \mathrm{d} \boldsymbol{k} v_{n n^{\prime}}(\boldsymbol{k}) \exp \left[\mathrm{i}\left(k_{x} \hat{X}-k_{y} \hat{P}\right)\right]
$$

The general term in the series is

$$
\hat{P}_{N}^{(n)}=\sum_{m} \sum_{n_{1}} \ldots \sum_{n_{N-1}} \sum_{m^{\prime}}|m\rangle\left\langle m^{\prime}\right| \frac{V_{m m_{1}}(\hat{X}, \hat{P}) V_{n_{1} n_{2}}(\hat{X}, \hat{P}) \ldots V_{n_{N-1} m^{\prime}}(\hat{X}, \hat{P})}{\left(h \omega_{c}\right)^{N}(n-m)\left(n-n_{1}\right) \ldots\left(n-m^{\prime}\right)}
$$

where exactly one of the integers $m, n_{1}, n_{2}, \ldots, m^{\prime}$ is equal to $n$ and the corresponding term is omitted from the product of $\left(n-n_{k}\right)$ terms in the denominator. Equations (3.10), (3.11) and (3.13) give the expansion of $\hat{P}^{(n)}$ in powers of $V$; because the Landau levels are all well separated there are no small denominators appearing in this series. 
Using equations (2.16) and (3.10)-(3.13) we can calculate the Hamiltonian and normalisation operators restricted to the $n$th Landau level (cf equation (3.4)) as a power series in $V$. The general term will not be given; the second term of each series is zero:

$$
\begin{aligned}
& \hat{H}^{(n) *}=\left\langle n\left|\hat{P}^{(n)} \hat{H} \hat{P}^{(n)}\right| n\right\rangle=\left(n+\frac{1}{2}\right) h \omega_{\mathrm{c}}+V_{n n}(\hat{X}, \hat{P})+\mathrm{O}\left(V^{3}\right) \\
& \hat{P}^{(n) *}=\left\langle n\left|\hat{P}^{(n)}\right| n\right\rangle=\hat{1}^{*}+\mathrm{O}\left(V^{3}\right) .
\end{aligned}
$$

From (3.15) we see that the normalisation operator, $\hat{P}^{(n) *}$, is non-singular for small enough values of $V$, as was assumed. Substituting these results into (3.6) we find that the first-order term of the exact effective Hamiltonian is given by (2.19) and again the second-order term vanishes:

$$
\hat{H}_{\mathrm{eff}}^{(n)}=\left(n+\frac{1}{2}\right) h \omega_{\mathrm{c}}+V_{n n}(\hat{X}, \hat{P})+\mathrm{O}\left(V^{3}\right)
$$

\section{Symmetries of the effective Hamiltonian}

The lowest order approximation to $\hat{H}_{\text {eff }}^{(n)}$ is given by (2.19). It is noteworthy that this approximate effective Hamiltonian retains all of the geometrical symmetries of the potential. If the potential has translational symmetry, the effective Hamiltonian has the same spatial frequencies in phase space as the potential has in real space, and therefore has the same translational symmetry. Also, the matrix element (2.18) which multiplies the Fourier coefficients of the potential only depends on the magnitude of the wavevector $\boldsymbol{k}$ ( since, in this case, $n=n^{\prime}$ ). The approximate effective Hamiltonian therefore retains the rotational and reflection symmetries of the potential. In this section it will be shown that the exact effective Hamiltonian also retains the translational and rotational symmetries of the potential, but not the reflection symmetries.

Because the effective Hamiltonian is represented as a Fourier integral, the Weyl symbol of $\hat{H}=H(\hat{x}, \hat{p})$ is $H(x, p)$ (see appendix 1 ). It will prove useful to define operators which have the effect of translating, rotating and reflecting the Weyl symbol of an operator. The translation operator in phase space is

$$
\hat{T}(X, P)=\exp [\mathrm{i}(P \hat{x}-X \hat{p}) / \hbar]
$$

If the operator $\hat{A}$ is represented by the Weyl symbol $A(x, p)$, then the Weyl symbol of the operator $\hat{T}(X, P) \hat{A} \hat{T}^{+}(X, P)$ is $A(x-X, p-P)$, i.e. it has been translated through the vector $(X, P)$. The translation operators have the composition law

$$
\hat{T}(X, P) \hat{T}\left(X^{\prime}, P^{\prime}\right)=\exp \left[\mathrm{i}\left(X^{\prime} P-X P^{\prime}\right) / 2 \hbar\right] \hat{T}\left(X+X^{\prime}, P+P^{\prime}\right)
$$

and the adjoint is given by $\hat{T}^{+}(X, P)=\hat{T}(-X,-P)$. The composition law (4.2) shows that the algebra of these phase space translation operators is the same as that of the magnetic translation operators (Brown 1964).

The rotation operator in phase space, which rotates the Weyl symbol of an operator through an angle $\theta$ about the origin, is

$$
\hat{R}(\theta)=\exp \left(\frac{1}{2 \hbar}\left(\hat{x}^{2}+\hat{p}^{2}+\hbar\right) \theta\right) .
$$

This is a special case of the symplectic transformation discussed in appendix 1 . The constant $\frac{1}{2} \hbar$ is added to the harmonic oscillator Hamiltonian in order that $\hat{R}(2 \pi)=\hat{1}$, 
instead of $-\hat{1}$ if this were omitted. The composition law for rotations is obvious: $\hat{R}(\theta) \hat{R}\left(\theta^{\prime}\right)=\hat{R}\left(\theta+\theta^{\prime}\right)$. The rule for combining rotations and translations is

$$
\hat{R}(\theta) \hat{T}(X, P)=\hat{T}\left(X^{\prime}, P^{\prime}\right) \hat{R}(\theta)
$$

where $\left(X^{\prime}, P^{\prime}\right)$ is the result of rotating the vector $(X, P)$ by the angle $\theta$. This result is not obvious; there could be a phase factor involved as in (4.2), but it follows from the results of appendix 1 , since $\hat{R}(\theta)$ is a symplectic transformation and $\hat{T}(X, P)$ is a function of a linear combination of $\hat{x}$ and $\hat{p}$. The operator $\hat{M}$ which produces a reflection about the line $p=0$ is defined by

$$
\left|\psi_{M}\right\rangle=\hat{M}|\psi\rangle \quad \psi_{M}(x)=\psi^{*}(x)
$$

i.e. we take the complex conjugate in the position representation.

The terms of order $V^{N}$ in the expansion of $\hat{H}_{\mathrm{eff}}^{(n)}$ contain products of $N$ operators $V_{m m^{\prime}}(\hat{x}, \hat{p})$, given by equation (3.12). The first and last labels are equal to $n$, the Landau level index, i.e. the product is of the form $\hat{V}_{n n_{1}} \hat{V}_{n_{1} n_{2}} \ldots \hat{V}_{n_{N-1} n}$, and the $n_{1} n_{2} \ldots$ are free to take any value except $n$. In order to understand the symmetry properties of the effective Hamiltonian it is necessary to consider the commutation relations of the $\hat{V}_{m m}$ operators with the symmetry operators $\hat{T}, \hat{R}$ and $\hat{M}$.

The operator $V_{m m}(\hat{x}, \hat{p})$ has the same Fourier coefficients as $u(\boldsymbol{r})$, so that if $u(\boldsymbol{r})$ is invariant under translation by a vector $\boldsymbol{R}$, the Weyl symbol of $V_{m m} \cdot(\hat{x}, \hat{p})$ is invariant under the action of the translation operator $\hat{T}(X, P)$, where $(X, P)=\boldsymbol{R}$. The operator $V_{n m}(\hat{x}, \hat{p})$ therefore commutes with $\hat{T}(\boldsymbol{R})$, where $\boldsymbol{R}$ is a lattice vector:

$$
V_{m m} \cdot(\hat{x}, \hat{p}) \hat{T}(\boldsymbol{R})=\hat{T}(\boldsymbol{R}) V_{m m}(\hat{x}, \hat{p}) \text {. }
$$

Any product of $\hat{V}_{m m}$ s therefore also commutes with $\hat{T}(\boldsymbol{R})$, so that the exact effective Hamiltonian does have the translational symmetry of the potential.

Next consider the effect of the rotation operator $\hat{R}(\theta)$ on $\hat{V}_{m m^{\prime}}$. From (3.12) and (4.1), we see that $\hat{V}_{m m^{\prime}}$ can be written in the form

$$
\hat{V}_{m m^{\prime}}=\int \mathrm{d} \boldsymbol{k} \exp \left[\mathrm{i}\left(m-m^{\prime}\right) \theta\right] f(k) \tilde{u}(\boldsymbol{k}) \hat{T}(\boldsymbol{k} \boldsymbol{h})
$$

where $(k, \theta)$ are polar coordinates of $\boldsymbol{k}$. Using (4.4), we see that if $u(\boldsymbol{r})$ is invariant under rotation by the angle $\theta$ about the origin, then

$$
\hat{R}(\theta) \hat{V}_{m m^{\prime}}=\exp \left[\mathrm{i}\left(m-m^{\prime}\right) \theta\right] \hat{V}_{m m^{\prime}} \hat{R}(\theta)
$$

so that only the diagonal terms $\left(m=m^{\prime}\right)$ commute with $\hat{R}(\theta)$. For all of the products of terms the first and last indices are equal to $n$, so that

$$
\begin{aligned}
\hat{R}(\theta) \hat{V}_{n n_{1}} \hat{V}_{n_{2} n_{2}} & \ldots \hat{V}_{n_{N-1} n}=\exp \left\{\mathrm { i } \left[\left(n-n_{1}\right)+\left(n_{1}-n_{2}\right)+\ldots\right.\right. \\
& \left.\left.+\left(n_{N-1}-n\right)\right]\right\} \hat{V}_{n n_{1}} \hat{V}_{n_{1} n_{2}} \ldots \hat{V}_{n_{N-1} n} \hat{R}(\theta)=\hat{V}_{n n_{1}} \hat{V}_{n_{1} n_{2}} \ldots \hat{V}_{n_{V-1} n} \hat{R}(\theta)
\end{aligned}
$$

and the exact effective Hamiltonian does indeed commute with $\hat{R}(\theta)$.

If the potential has reflection symmetry, again $\hat{M}$ commutes with the diagonal terms, $\hat{V}_{m m}$, but not with the off-diagonal terms $\hat{V}_{m m^{\prime}}$. In this case however there is no reason to expect that the products of off-diagonal $\hat{V}_{m m^{\prime}}$ commute with $\hat{M}$ and it is easy to find cases where they do not. The exact effective Hamiltonian does not therefore preserve reflection symmetries of the potential beyond the lowest order term. This is perhaps not unexpected, since applying a magnetic field imposes a chirality on the system. 


\section{Conclusions}

The influence of a scalar potential $V(x, y)$ on a Landau level in two dimensions can be represented by a one-dimensional effective Hamiltonian $H_{\text {eff }}^{(n)}(x, p)$, which remains valid provided the gaps between the $n$th Landau levels remain open and the normalisation operator $\hat{P}^{(n) *}$ (equation (3.15)) remains non-singular. The effective Hamiltonian can be expressed as a power series in $V / E_{c}$. The lowest order term was derived in $\S 2$ (equation (2.19)), and the higher-order terms can be written down using the results of $\S 3$. The second-order term in this expansion is identically zero (equation (3.16)).

This representation is useful because the original two-dimensional problem has been reduced to one dimension and because the phase space function $H_{\text {eff }}^{(n)}(x, p)$ resembles the potential $V(x, y)$. When the ratio $\beta$ of the area of a flux quantum to the characteristic area of the potential is small, $H \rightarrow V$ (equation (2.21)). Also, the results of $\S 4$ show that $H$ preserves the translational and rotational symmetries of $V$ exactly, even when $\beta$ is not small, but only retains the reflection symmetries of $V$ in the lowest order of the perturbation expansion in powers of $V / E_{c}$. Numerical experiments on effective Hamiltonians periodic in $x$ and $p$ show that the spectrum is of an unusual type when the effective Hamiltonian has exact threefold or fourfold rotational symmetry in phase space (Wilkinson 1984). The results of this paper show that this symmetry corresponds to natural crystallographic symmetries of the periodic potential.

\section{Acknowledgments}

I acknowledge financial support from a Weingart Fellowship of the California Institute of Technology. This work was not supported by any military agency.

\section{Appendix 1}

Canonical transformations are used in the derivation of the effective Hamiltonian and the symmetry operators introduced in $\$ 4$ are all canonical transformations. This appendix will introduce the Weyl symbol as a way of representing operators as functions in phase space and will discuss its most important property, which guarantees that a certain type of classical canonical transformation can be given an unambiguous meaning in quantum mechanical problems. All the canonical transformations required in this paper are of this type.

The Weyl symbol $A_{\mathrm{W}}(x, p)$ of an operator $\hat{A}$ is defined by the equations

$$
\begin{aligned}
& A_{\mathrm{W}}(x, p)=\operatorname{Tr}[\hat{W}(x, p) \hat{A}] \\
& \hat{W}(x, p)=\frac{\hbar}{2 \pi} \iint \mathrm{d} X \mathrm{~d} P \exp \left(\frac{\mathrm{i}}{\hbar}[(p-\hat{p}) P+(x-\hat{x}) X]\right) .
\end{aligned}
$$

The motivation for this definition comes from the fact that the Weyl symbol $A_{\mathrm{W}}(x, p)$ transforms classically under the action of a linear canonical transformation

$$
\left(\begin{array}{l}
x^{\prime} \\
p^{\prime}
\end{array}\right)=\tilde{M}\left(\begin{array}{l}
x \\
p
\end{array}\right) .
$$


This statement needs some explanation. A symplectic transformation of the form (A1.3) is generated by a quadratic Hamiltonian $\hat{H}$ acting over a time $t$

$$
\hat{H}=\frac{1}{2}\left[A \hat{x}^{2}+B \hat{p}^{2}+C(\hat{x} \hat{p}+\hat{p} \hat{x})\right]
$$

and the operator $\hat{A}$ transforms as follows:

$$
\hat{A}(t)=\hat{U}^{+}(t) \hat{A} \hat{U}(t) \quad \hat{U}(t)=\mathrm{e}^{-i \hat{H} t} .
$$

The result is that the Weyl symbol of $\hat{A}(t)$ evolves classically:

$$
\partial A_{\mathrm{W}}(t) / \partial t=\left\{A_{\mathrm{W}}, H\right\}
$$

where \{\} is the Poisson bracket. A derivation of this result has been given by Ozorio de Almeida and Hannay (1982).

The other important point is that if the operator $\hat{A}$ is a function of a linear combination of $\hat{x}$ and $\hat{p}$

$$
\hat{A}=A(\alpha \hat{x}+\beta \hat{p})
$$

then the Weyl symbol of $\hat{A}$ is simply $A(\alpha x+\beta p)$. Similarly, if $\hat{A}$ is a sum of functions of this type, the Weyl symbol is also obtained by replacing $\hat{x}, \hat{p}$ by the $c$ numbers $x$, p. Thus, if $\boldsymbol{A}(\hat{\boldsymbol{x}}, \hat{p})$ is expressed as a Fourier integral

$$
\hat{A}=A(\hat{x}, \hat{p})=\iint \mathrm{d} \boldsymbol{k} \tilde{A}(\boldsymbol{k}) \exp \left[\mathrm{i}\left(k_{x} \hat{x}+k_{y} \hat{p}\right)\right]
$$

then the Weyl symbol of $\hat{A}$ is $A_{\mathrm{W}}(x, p)=A(x, p)$. Provided we express all operators as a Fourier integral, they transform classically under linear canonical transformations.

\section{Appendix 2}

This appendix gives the derivation of equation (2.18). To simplify the notation, we will work in units where $[\hat{x}, \hat{p}]=\mathrm{i}$ and evaluate the matrix element

$$
I_{n n^{\prime}}(\alpha, \beta)=\left\langle n|\exp [\mathrm{i}(\alpha \hat{x}+\beta \hat{p})]| n^{\prime}\right\rangle
$$

where $|n\rangle,\left|n^{\prime}\right\rangle$ are eigenstates of the harmonic oscillator Hamiltonian.

It is convenient to represent $(\alpha, \beta)$ by a single complex number $z$; define

$$
z=\frac{\beta+\mathrm{i} \alpha}{\sqrt{2}} \quad \hat{T}(z)=\exp \left(z \hat{a}-\bar{z} \hat{a}^{+}\right)
$$

where $\hat{a}^{+}, \hat{a}$ are the usual annihilation and creation operators, so that

$$
I_{n n^{\prime}}(z)=\frac{1}{\left(n ! n^{\prime} !\right)^{1 / 2}}\left\langle 0\left|\hat{a}^{n} \hat{T}(z) \hat{a}^{+n}\right| 0\right\rangle .
$$

The first step in evaluating (A2.3) is to express it in terms of matrix elements of the form $\left\langle 0\left|\hat{a}^{k} \hat{T}(z)\right| 0\right\rangle$. It is easy to show that

$$
\left[\hat{T}(z), \hat{a}^{+}\right]=z \hat{T}(z)
$$


so that

$$
\begin{aligned}
I_{n n^{\prime}}(z) & \left.=\frac{1}{\left(n ! n^{\prime} !\right)^{1 / 2}}\langle 0| \hat{a}^{n} \hat{a}^{+}+z\right)^{n^{\prime}} \hat{T}(z)|0\rangle \\
= & \frac{1}{\left(n ! n^{\prime} !\right)^{1 / 2}} \sum_{m=0}^{n^{\prime}} \frac{n^{\prime} !}{\left(n^{\prime}-m\right) ! m !} z^{n^{\prime}-m}\left\langle 0\left|\hat{a}^{n} \hat{a}^{+m} \hat{T}(z)\right| 0\right\rangle \\
= & \left(n ! n^{\prime} !\right)^{1 / 2} \sum_{m=0}^{n^{\prime}} \frac{1}{\left(n^{\prime}-m\right) !(n-m) ! m !} z^{n^{\prime}-m}\left\langle 0\left|\hat{a}^{n-m} \hat{T}(z)\right| 0\right\rangle
\end{aligned}
$$

where in the final step we have assumed that $n \geqslant n^{\prime}$.

The next step is to evaluate the matrix elements $\left\langle 0\left|\hat{a}^{k} \hat{T}(z)\right| 0\right\rangle$. Using the BakerHausdorf formula, we find

$$
\hat{T}(z)=\mathrm{e}^{z \hat{a}} \mathrm{e}^{-\bar{z} \hat{a}^{+}} \mathrm{e}^{-z \bar{z} / 2}
$$

so that

$$
\begin{aligned}
\left\langle 0\left|\hat{a}^{k} \hat{T}(z)\right| 0\right\rangle & =\mathrm{e}^{z \bar{z} / 2} \sum_{m} \sum_{m^{\prime}} \frac{1}{m ! m^{\prime} !} z^{m}(-\bar{z})^{m^{\prime}}\left\langle 0\left|\hat{a}^{k+m} \hat{a}^{+m^{\prime}}\right| 0\right\rangle \\
& =\mathrm{e}^{z \bar{z} / 2} \sum_{m} \frac{1}{(m+k) !} \frac{1}{m !}(-z \bar{z})^{m}(-\bar{z})^{k}\left\langle 0\left|\hat{a}^{m+k} \hat{a}^{+(m+k)}\right| 0\right\rangle \\
& =(-\bar{z})^{k} \mathrm{e}^{-z \bar{z} / 2} .
\end{aligned}
$$

From (A2.5) and (A2.7), we have

$$
I_{n n^{\prime}}(z)=\left(\frac{n^{\prime} !}{n !}\right)^{1 / 2}(-\bar{z})^{n-n^{\prime}} \mathrm{e}^{-z \bar{z} / 2} L_{n^{\prime}}^{\left(n-n^{\prime}\right)}(z \bar{z})
$$

where

$$
L_{n^{\prime}}^{\left(n^{\prime}-n\right)}(x)=\sum_{m=0}^{n^{\prime}} \frac{n !}{\left(n-n^{\prime}+m\right) !(n-m) ! m !}(-x)^{m}
$$

is an associated Laguerre polynomial (Abramovitz and Stegun 1964). Inserting the correct dimensional factors in (A2.8) we obtain (2.18). If $n^{\prime}>n$, we can calculate $I_{n n^{\prime}}$ using the relation

$$
I_{n n^{\prime}}(z)=\bar{I}_{n^{\prime} n}(-z)
$$

\section{References}

Abramovitz M and Stegun I A 1964 Handbook of Mathematical Functions (Washington, DC: National Bureau of Standards)

Brown E 1964 Phys. Rev. 133 A1038-44

Claro F and Wannier G H 1979 Phys. Rev. B $196068-74$

Hofstadter D R 1976 Phys. Rev. B 14 2239-49

Ozorio de Almeida A M and Hannay J H 1982 Ann. Phys., NY 138 115-34

Rauh A 1974 Phys. Status Solidi b 65 K131-5

1975 Phys. Status Solidi b 69 K9-13

Schellnhuber H J, Obermair G M and Rauh A 1981 Phys. Rev. B 23 5191-202

Simon B 1982 Adv. Appl. Math. 3 463-90

Wilkinson M 1984 Proc. R. Soc. A 391 305-50 